Cell Research (1997), 7, 31-38

\title{
Cytological identification of an isotetrasomic in rice and its application to centromere mapping ${ }^{1}$
}

Cheng Zhu Kuan, Heng Xiu YU, Chang Jie Yan, Li Huang ZHU*, Ming Hong GU

Department of Agronomy, Agriculture College, Yangzhou University, Yangzhou 225009

* Institute of Genetics, Chinese Academy of Sciences, Beijing 100101

\section{ABSTRACT}

The aneuploid with isochromosome or telochromosome is ideal material for exploring the position of centromere in lingkage map. For obtaining these aneuploids in rice, the primary trisomics from triplo- 1 to triplo- 12 and the aneuploids derived from a triploid of indica rice variety Zhongxian 3037 were carefully investigated. From the offsprings of triplo-10, a primary trisomic of chromosome 10 of the variety, an isotetrasomic "triplo- 10- 1" was ohtained. Cytological investigation revealed that a pair of extra isochromosomes of triplo-10-1 were come from the short arm of chromosome 10. In the offsprings of the isotetrasomic, a secondary trisomic "triplo-10-2", in which the extra- chromosome was an isochromosome derived from the short arm of chromosome 10, was identified. With the isotetrasomic, secondary trisomic, primary trisomic and diploid of variety Zhongxian 3037, different molecular markers were used for exploring the position of the centromere of chromosome 10. Based on the DNA dosage effect, it was verified that the molecular markers Gl125, G333 and L169 were located on the short arm, G1084 and other 16 available molecular markers were on the long arm of chromosome 10. So the centromere of chromosome 10 was located somewhere betweenG1125 and G1084 according to the RFLP linkage map given by Kurata et al[1]. The distance from G1125 to G1084 was about 3.2cM.

Key words: Rice, isotetrasomic, centromere mapping.

1. Dedicated to 80th birthday of Professor Lu Ji SHI. 
Isotetrasomic of rice and centromere mapping

\section{INTRODUCTION}

Some aneuploids of rice, such as primary trisomics, have been available for rice geneticists[2, 3]. In last two decades, a number of genes affecting different agronomic characteristics were located on rice chromosomes through the use of primary trisomics. The corresponding relationship between chromosomes and linkage groups was reported by Khush et al. and Iwata et al. using different primary trisomics. However, development of other aneuploids is also desirable for genome study in rice. Secondary trisomic, in which the extra chromosome is an isochromosome, has been found in maize[4], tomato[5], and other plants. With the aid of secondary trisomic, centromere mapping has been successfully conducted on these crops. Isotetrasomic is another aneuploid in which two extra chromosomes are a pair of isochromosomes. It has unique advantage for centromere mapping due to the dosage effect of its extra arms. But few researches have been made in this area.

Variations related to centromere such as secondary trisomics are usually originated from misdivision of centromere during meiosis, and the misdivision often occurs in univalent chromosomes. For obtaining such kinds of variations, all the 12 primary trisomics and other aneuploids derived from a triploid of Zhongxian 3037, a variety of indica rice, were planted in 1994. Cytological investigation was carefully conducted for all the morphological variations occurred in their progenies. A variation with 26 chromosomes in somatic cell was found in the progenies of triplo-10. The investigation on chromosomes at pachytene stage of meiosis indicated that the two extra chromosomes of the variation were a pair of isochromosomes consisting of the short arm of chromosomes 10. A secondary trisomic plant was obtained from the progeny of this isotetrasomic after selfing. Present paper is focused on the cytological identification of the aneuploids and the application of them to mapping the centromere of chromosome 10.

\section{MATERIALS AND METHODS}

\section{Slide preparation and chromosome checking}

12 primary trisomics and other aneuploids obtained from a triploid of variety Zhongxian 3037 were planted in the field in 1994. All variations morphologically different from the original trisomic and diploid were marked and the young panicles of these plants were harvested at meiosis stage and fixed in 3:1 Carnoy solution to which $0.5 \% \mathrm{FeCl}_{3}$ was added. Slide preparation for chromosome checking at pachytene stage of meiosis were conducted according to Wu's method[6].

\section{Southern analysis}

RFLP markers designated as RG\#, RZ\#, and CDO\# were kindly provided by Dr. S.D. Tanksley from Cornell University, USA, and probes of G\#, L\#and C\# were obtained from the MAFF DNA Bank at NIAR, Japan. Southern blots for dosage effect analysis were prepared as described by McCouch et al[7]. Genomic DNA was extracted from young leaves, and then digested with the restriction enzyme EcoRI. The amount of DNA from each plant was carefully controlled to be exactly $5 \mu \mathrm{g}$ and aliquoted to each lane in a gel. Furthermore, large amonts of digests were prepared, and several filters were made for each digestion batch to assure uniformity among filters. 
Hybridization was according to Bernatzky and Tanksley[8]. Whole plasmid hexamers were labeled with ${ }^{32} \mathrm{P}$-dCTP, and used as probe on filters of rice DNA. After hybridization filters were washed at $65{ }^{\circ} \mathrm{C}$ for $10 \mathrm{~min}$ at $2 \times \mathrm{SSC}, 1 \times \mathrm{SSC}$ and $0.5 \mathrm{SSC}$ (all washing solutions used containing 0.1 $\% \mathrm{SDS}$ ), and exposed to X-ray films with intensifier screens.

\section{RESULTS}

\section{Morphological characteristics of the variations}

The plant of triplo- 10 is significantly different from diploid plant (Plate I, A, B). In general, it has more tillers with thinner stems, shorter plant height and smaller kernels (the width of the kernel is about $2 / 3$ of that of the diploid). In most cases a few awns occurred at the top kernels of panicle and small seeds can be served as the symbol of triplo-10 in rice variety Zhongxian 3037. From the progenies of triplo- 10, a variation designated as triplo-10-1 was found. It showed much lower fertility (7.3 $\%$ seed setting) compared with normal triplo -10 (about $57.5 \%$ ). Besides, the plant had shorter stature than the normal triplo- 10 with dark green leaves in most cases. Next year, we found another variation which showed much higher fertility $(68.3 \%$ seed setting) with more tillers than the original plant (Plate I, C). The plant was designated as triplo-10-2.

\section{Cytological investigation on the variation plants}

Cytological investigation was conducted on both triplo-10-1 and triplo -10-2. Triplo-10-1 showed 26 chromosomes with four synaptic configurations at diakinesis and metaphase I in meiosis, i.e., 13 II, 11 II + 1 IV, 11 II + 1 III + 1 I or 12 II + 2 I. Its two extra chromosomes could pair with each other, or pair with other chromosolnes forming trivalent and tetravalent. It seems that triplo-10-1 might be an isotetrasomic plant. With the extra chromosomes being often linked with nucleoli, the aneuploid plant could be identified as a tetrasomic of a nucleolar chromosome. Cytological investigation showed that Zhongxian 3037 has two nucleolar chromosomes, i.e., chromosome 9 and 10 respectively according to the numbering system reported on the Second International Rice Genetics Symposium in 1990[9]. (They are chromosome 10 and 12 respectively according to Cheng and Gu[10]). Among them, the longer one is chromosome 9 and the shorter one is chromosome 10. Further investigation revealed that self- pairing between the two arms of the extra chromosomes frequently occurred in the formation of univalent and often linked with a nucleolus (Plate I, D). The two extra chromosomes could also pair with each other, forming a bar or a circle shaped configulation (Plate I, F, G). Sometimes, it was found that one of the self-paired isochromosome was linked with the short arm of chromosome 10 (shorter nucleolar chromosome) or directly connected with the centromere of the chromosome (Plate I, H, I), while the other self-paired isochromosome was still linked with imcleoli. Another configuration observed frequently was the one in which the two self-paired isochromosomes were all linked with the short arm of chromosolne 10 forming a Y-type configuration near the nucleolus (Plate I, J). All 
Isotetrasomic of rice and centromere mapping
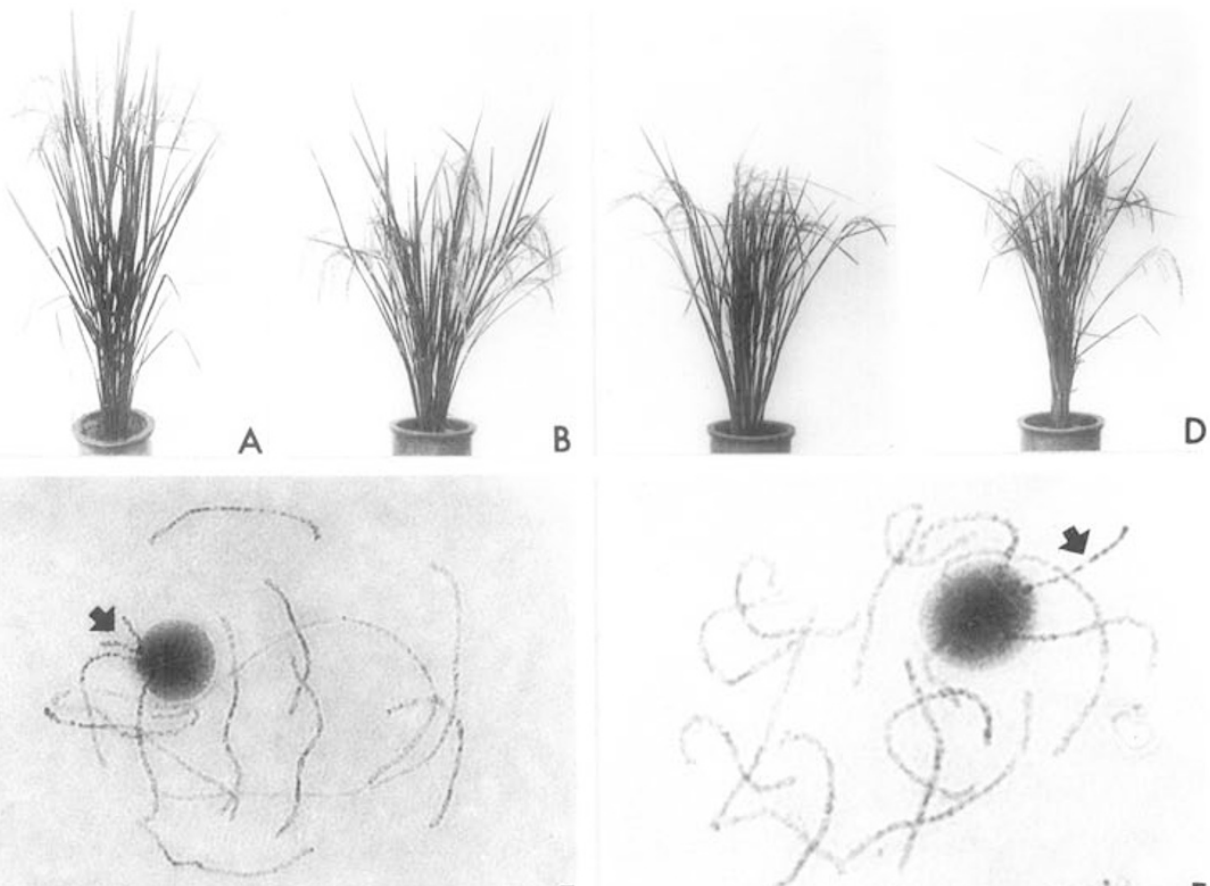

E
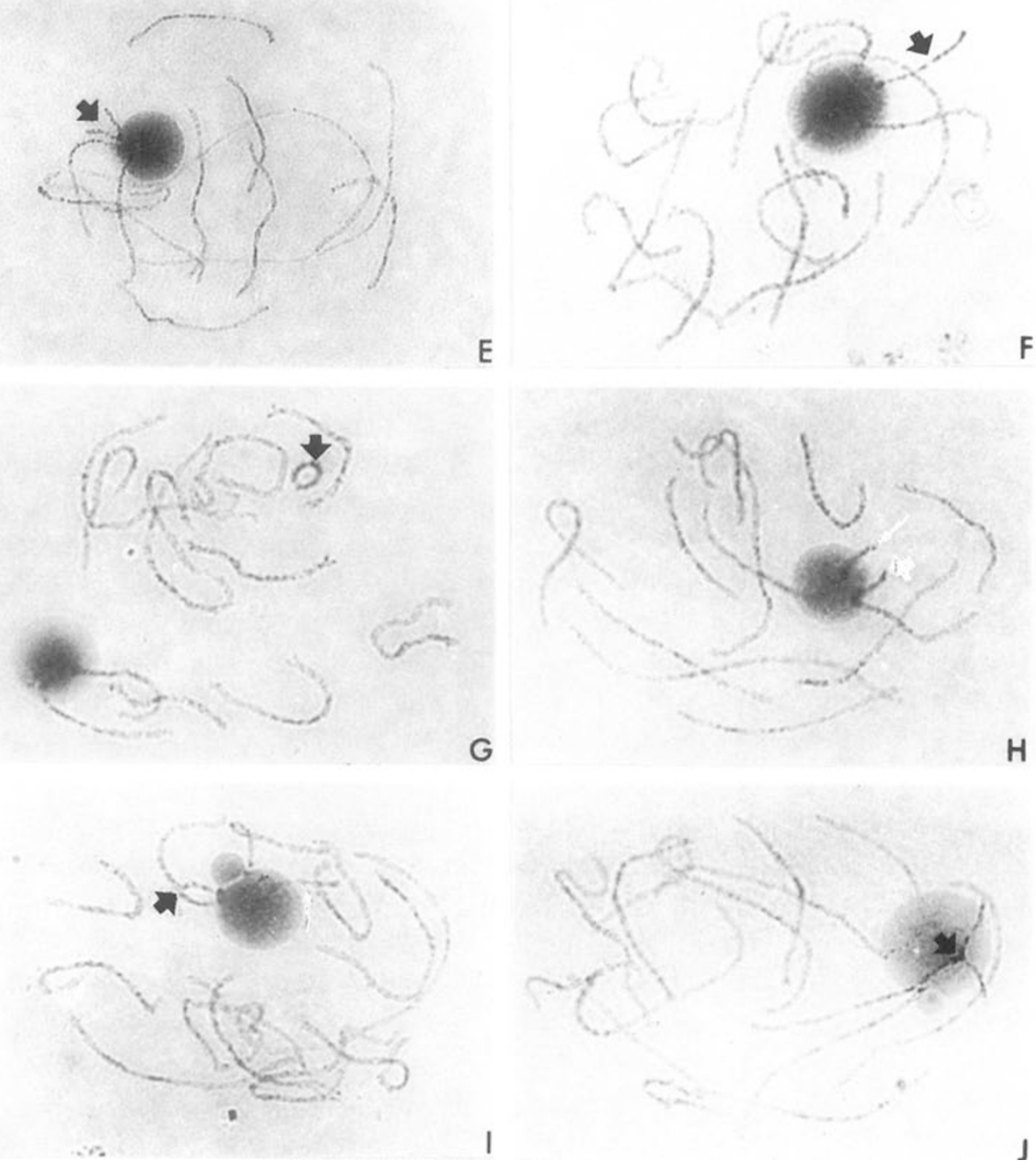
Cheng ZK et al.
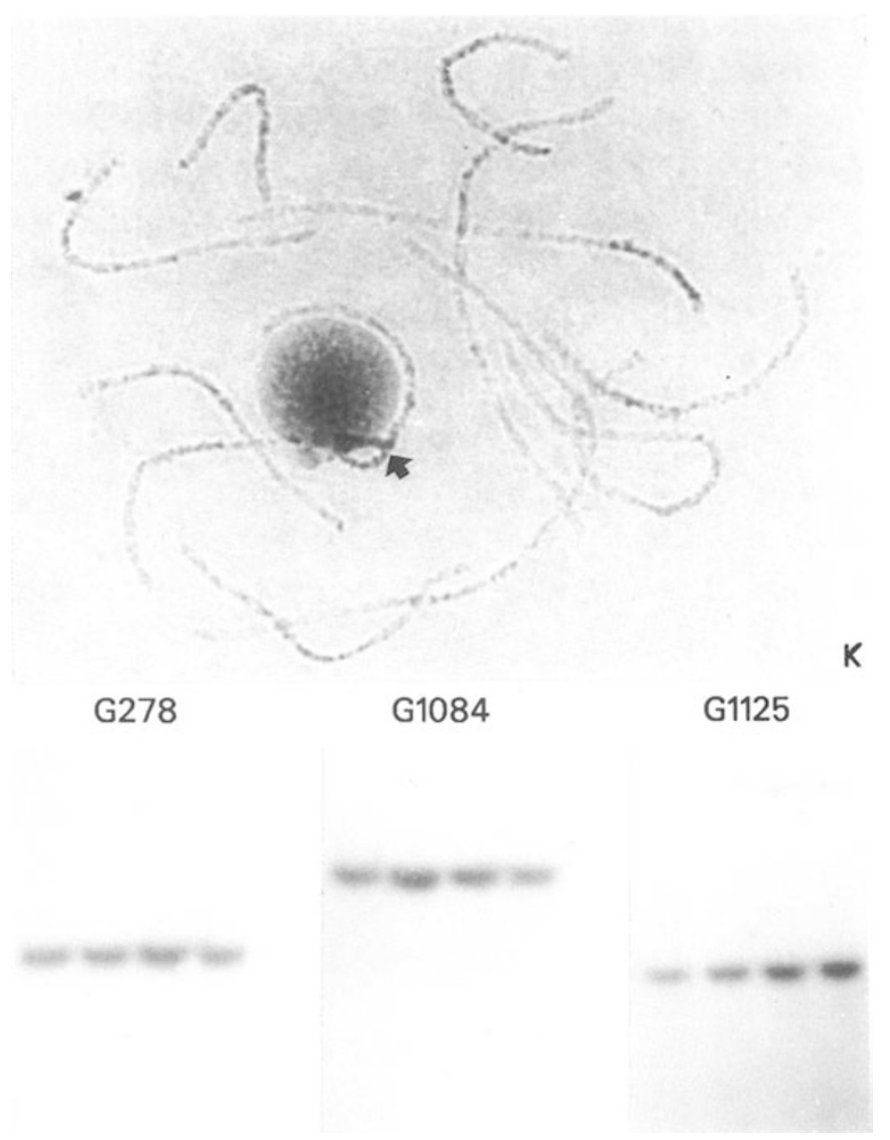

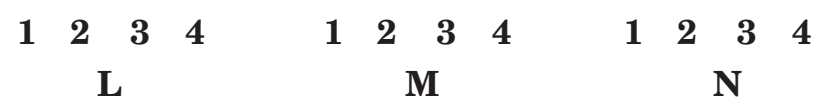

\section{Explanation of plates}

A. A plant of Zhongxian 3037. B. A plant of Zhongxian 3037 triplo-10. C. A plant of the isotetrasomic. D. A plant of the secondary trisomic. E-J. Pachytene chromosomes of an isotetrasomic. E. The triangle shows the univalent of isochromosomes linked with a nucleolus. F. The triangle shows the two isochromosomes forming a bar linked with a nucleolus. G. The triangle shows the circular type bivalent of the isochromosomes linked with a nucleolus. H. The triangle shows one self- paired isoromosome linked with the short arm of chromosome 10 . The arrow shows another self-paired isochromosome still linked with a nucleus. I. The triangle shows one self-paired isochromosome linked with chromosomes 10 at centromere position. The arrow shows another self- paired isochromosome still attached to a nucleolus. J. The triangle shows the two univalents of the isochromosomes linked with the short arm of chromosome 10 forming a Y-type configuration. K. Pachytene chromosomes of a secondary trisomic. The triangle shows the selfpaired isochromosome linked with chromosome 10 at centromere position and also with a nucleolus. L-N. Autoradiograms derived from three hybridizations with different clones in the same filter.

1. DNA of diploid Zhongxian 3037. 2. DNA of triplo-10. 3. DNA of the secondary trisomic. 4. DNA of the isotetrasomic. 
the evidence on both synaptic configurations and the similarity in length and chromomere distribution between the univalents self- paired and the short arm of chromosome 10 in bivalent indicates that the two extra chromosomes of triplo- 10-1 are the isochromosomes derived from the short arm of chromosome 10. It is therefore concluded that triplo-10-1 is an isotetrasomic plant with respect to the short arm of chromosome 10.

On the other hand, cytological investigation showed that the variant plant of triplo-10-2 had 25 chromosomes in somatic cells, the extra chromosome of the variation was an isochromosome. At paehytene stage of PMC, one end of the extra chromosome formed after self-pairing was always linked to the centromere of chromosome 10, forming a Y-type configuration while the other end was connected to the nucleolus (Plate II, K). The synaptic pattern indicates that triplo-10-2 is a secondary trisomic in which the extra chromosome is originated from the short arm of chromosome 10 .

\section{Centromere mapping of chromosome 10}

In recent years remarkable achievements have been made in RFLP mapping on both molecular markers and genes important to expression of agronomic traits in rice[1, 11]. But few papers reported studies on the centromere position on rice[12].

For centromere mapping, the isotetrasomic and secondary trisomic mentioned above are very useful since the amount of DNA provided by the short arm of chromosome 10 within the diploid, triplo-10, secondary trisomic and isotetrasomic would be in the proportion of $2: 3: 4: 6$. It can be detected through dosage effect after molecular hybridization in Southern analysis if the markers are located on the short arm of chromosome 10 .

DNA extracted from leaves of diploid Zhongxian 3037, triplo-10, triplo-10-2 and triplo-10-1 was digested with EcoR I respectively. Equal amount of digested DNA from each sample was loaded on the gel for the comparison of DNA dosage in Southern analysis with different probes. Identical degree of darkness on the autoradiograms was found when the clone G278 located on chromosome 8 was hybridized with the filter (Plate II, L), which proved that the amounts of DNA added to the four lanes were approximately equal. When other 20 clones located on chromosome 10 were hybridized to the filters, 17 clones, i.e., C16, C223, G37, G89-2, G127, G291, G1082, G1084, G2155, RG241, RG257, RG561, RG752, RZ561, RZ81l, CDO94 and CDO250, showed higher dosage in triplo-10 as compared with the diploid, secondary trisomic and isotetrasomic (Plate II, M). This indicated that these clones were on the long arm of chromosome 10. The other 3 clones, i.e., L169, G333 and G1125, showed perfect dosage effect as expected. The darkness varied proportionally to the copy number of the short arms of chromosome 10, indicating that all the 3 clones were located on short arm (Plate II, N). The closest markers available which show different results in dosage effect are G1084 and G1125 in the molecular linkage map 
developed by Kurata et al[1]. The data demonstrates that the centromere of chromosome 10 is located somewhere between these two markers and the distance between them is about $3.2 \mathrm{cM}$. Since there are other markers assigned to the segment between G1084 and Gl125, further investigation on the precise location of the centromere is needed. It is noted that all the markers on the linkage map of chromosome 10 according to Causse et al[11] are positioned on the long arm. RG257 located at one end and CDO94 located at the other end. It means that the linkage relationship presented in the map provids genetic information on long arm only. The distribution of the markers on chromosome 10 is shown in Fig 1 according to Kurata et al[1] and Causse et al[11]. It is hard to compare them with each other due to the lack of common markers in Fig 1.1 and Fig 1.2. Fig 1.3 is a map of chromosome 10 constructed in our Lab. with a $\mathrm{DH}$ population originating from $\mathrm{F} 1$ of Nanjing 11 (indica)/Balilla(japonica). From the map, both the centromere position detected by marker G1084 and others and the end of the long arm detected by CDO94, C16 ect. can be clearly identified. Comparing the common markers in Fig 1.2 and Fig 1.3,

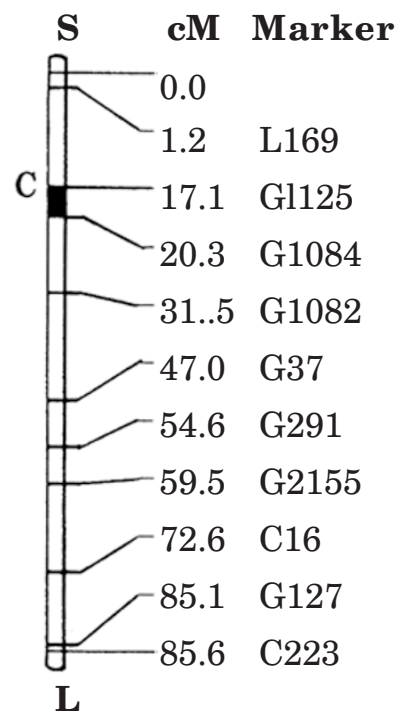

1
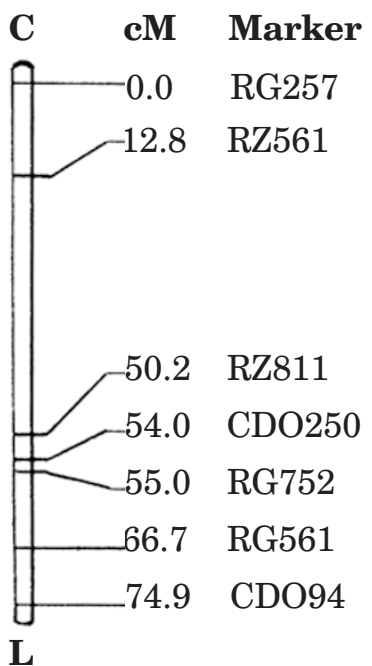

2

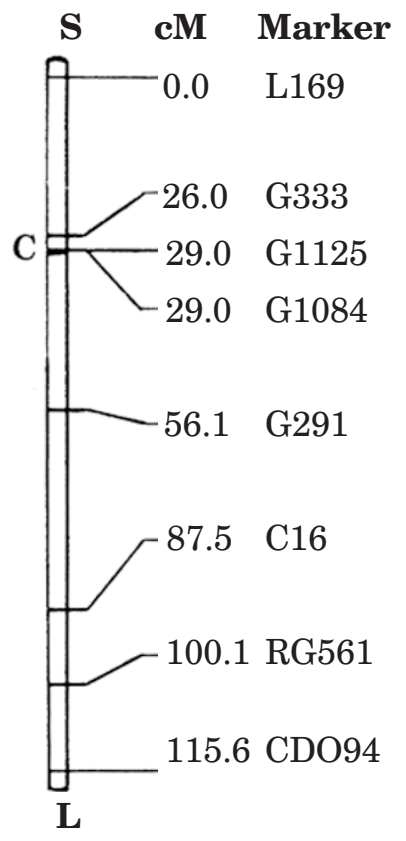

3

Fig 1. Distribution of molecular markers in chromosome 10 given by different researchers 1. The map according to by Kurata et al. 2. The map according to by Causse et al. 3. The map based on the $\mathrm{DH}$ population of Nanjing $11 /$ Balilla $_{1}$.

S: The shorter arm. L: The longer arm. C: The centromere. 
it can be found that CDO94 is located at a position close to the end of the long arm while RG561 is situated at the centromere side on the arm. So it can be concluded that RG257 is located in a position even closer to the centromere on the long arm while CDO94 is close to the end of the long arm of chromosome 10 in the map given by Causse et al[11].

\section{CONCLUSIONS}

Genetic stocks related to isochromosome or telochromosome are of prime importance for determining the position of the centromere and the orientations of linkage groups. Up to now, few reports are related to isochromosome or telochromosome in rice due to their limited size. In the present study both isotetrasomic and secondary trisomic of the short arm of chromosome 10 have been identified in the offsprings of the primary trisomic derived from a triploid plant of rice variety Zhongxian 3037. By studying the isotetrasomic, secondary trisomic and primary trisomic, the centromere of chromosome 10 has been positioned somewhere between G1084 and G1125 in the map given by Kurata et al[1]. The result was also verified by the RFLP lingkage map constructed in authors' Lab. with a $\mathrm{DH}$ population.

\section{REFERENCES}

[1] Kurata, N. et al. A 300 kilobase interval genetic map of rice including 883 expressed sequences. Nature Genetics 1994; 8:365-72.

[2] Iwata N, et al. Studies on the trisomics in rice plants Oryza sativa L. V. Relationship between the twelve chromosomes and the linkage groups. Jpn J Breed 1984; 34:314-21.

[3] Khush GS. et al. Origin, morphology, cytology and use in linkage mapping. Genetics 1984; 107:141-63.

[4] Rhoades MM. Studies of a telocentric chromosome in maize, with reference to the stability of its centromere. Genetics 1940: 25:483-520.

[5] Khush GS, CM Rick. Tomoto telotrisomics: origin,identification, and use in linkage mapping Cytologia 1968; 33:137-48.

[6] Wu HK. Note on preparing of pachytene chromosomes by double mordant. Scientific Agriculture 1967; 15:40-4.

[7] McCouch SR, et al. Molecular mapping of rice chromosome. Theor Appl Genet 1988; 76:815-29.

[8] Bernatzky R, Tanksley SD. Majority of random cDNA clones correspond to single loci in the tomato genome. Mol Gen Genet 1996; 203:8-14.

[9] Khush GS, T Kinoshita. Rice karyotype, marker genes, and linkage groups. In: Rice Biotechnology. IRRI press (Philippines), 1991:pp83-108.

[10] Cheng ZK, MH Gu. Karyotype analysis for pachytene chromosome of indica, japonica rice and their hybrid. Chinese J Genet 1994; 21:181-90.

[11] Causse Met al. Saturated molecular map of rice genome based on an interspecific backcross population. Genetics 1994; 138:1251-74.

[12] Singh $\mathrm{K}$ et al. Centromere mapping and orientation of the molecular linkage map of rice (Oryza sativa L.). Proc Natl Acad Sci USA 1996; 93:6163-8.

Received 25-12-1996 Revised 6-5-1997. Accepted 29-5-1997. 